\title{
Regularity for nonlinear evolution variational inequalities with delay terms
}

Hyun-Hee Rho and Jin-Mun Jeong*

${ }^{*}$ Correspondence:

jmjeong@pknu.ac.kr

Department of Applied

Mathematics, Pukyong National

University, Busan, 608-737, Korea

\begin{abstract}
In this paper, we deal with the regularity and a variation of constant formula for solutions of the nonlinear differential equation with delay nonlinear terms governed by the variational inequality in Hilbert spaces. Without the conditions of the uniform boundedness of the nonlinear terms and the compactness of the principal operators, we obtain the wellposedness and the norm estimate of the given equation by converting the problem into the contraction mapping principle on state space.

MSC: Primary 35K85; secondary 35F25
\end{abstract}

Keywords: variational inequality; subdifferential operator; delay term; regularity; analytic semigroup

\section{Introduction}

Let $H$ and $V$ be two complex Hilbert spaces. Assume that $V$ is dense subspace in $H$ and the injection of $V$ into $H$ is continuous. The norm on $V$ and $H$ will be denoted by $\|\cdot\|$ and $|\cdot|$, respectively. Let $A$ be a continuous linear operator from $V$ into $V^{*}$ which is assumed to satisfy Gårding's inequality, and let $\phi: V \rightarrow(-\infty,+\infty]$ be a lower semicontinuous, proper convex function. Then we study the following variational inequality problem with nonlinear term:

$$
\left\{\begin{array}{l}
\left(x^{\prime}(t)+A x(t), x(t)-z\right)+\phi(x(t))-\phi(z) \\
\quad \leq\left(\int_{-r}^{0} h(t, s, x(t), x(t+s), u(t)) \mu(d s)+k(t), x(t)-z\right), \quad \text { a.e. } \forall z \in V, \\
x(0)=g^{0}, \quad x(s)=g^{1}(s), \quad-r \leq s \leq 0 .
\end{array}\right.
$$

Here, $g=\left(g^{0}, g^{1}\right) \in H \times L^{2}(0, T ; V)$, a forcing term $k \in L^{2}\left(0, T ; V^{*}\right)$ and $h: \mathbb{R}^{+} \times V \times H \rightarrow H$ is a nonlinear mapping.

By the definition of the subdifferential operator $\partial \phi$, the problem (NVE) is represented by the following nonlinear functional differential problem:

$$
\left\{\begin{array}{l}
x^{\prime}(t)+A x(t)+\partial \phi(x(t)) \ni \int_{-r}^{0} h(t, s, x(t), x(t+s), u(t)) \mu(d s)+k(t), \quad 0<t, \\
x(0)=g^{0}, \quad x(s)=g^{1}(s), \quad-r \leq s \leq 0 .
\end{array}\right.
$$

The theory of variational evolution inequalities is one of the most important domains of application of the ideas and techniques of differential equations associated with maximal monotone operators and semigroups of nonlinear contractions. There is extensive

\section{Springer}

(c) 2014 Rho and Jeong; licensee Springer. This is an Open Access article distributed under the terms of the Creative Commons Attribution License (http://creativecommons.org/licenses/by/2.0), which permits unrestricted use, distribution, and reproduction in any medium, provided the original work is properly cited. 
literature on parabolic variational inequalities and the Stefan problems (see Lions [1, 2], Brézis [3, 4], Friedman [5], Elliott and Ockendon [6], Barbu [7, 8]). For more details on the applications of the theory we refer to the survey of Lions [9] and the book by Duvaut and Lions [10].

In this paper we are primarily interested in the regular problem that arise as direct consequences of the general theory developed previously, and we consider to put in perspective those models of initial value problems which can be formulated as nonlinear differential equations of variational inequalities. When the nonlinear mapping $h$ is a Lipschitz continuous from $\mathbb{R} \times V$ into $H$, we will find that the most part of the regularity for parabolic variational inequalities can also be applicable to (NDE) with nonlinear perturbations. The above operator $h$ is the semilinear case of the nonlinear part of quasilinear equations considered by Yong and Pan [11]. The approach used here is similar to that developed in [1214] on the general semilinear evolution equations.

Without conditions of the uniform boundedness of the nonlinear terms and the compactness of the principal operators, we obtain the wellposedness of (NDE) by converting the problem into the contraction mapping principle and the norm estimate of a solution of the above nonlinear equation on $L^{2}(0, T ; V) \cap W^{1,2}\left(0, T ; V^{*}\right) \cap C([0, T] ; H)$. Consequently, in view of the monotonicity of $\partial \phi$ and using the interpolation theory, we show that the solution mapping

$$
\begin{aligned}
H \times L^{2}(-r, 0 ; V) \times L^{2}(0, T ; H) & \ni\left(g^{0}, g^{1}, k\right) \mapsto x \\
& \in L^{2}(0, T ; D(A)) \cap W^{1,2}(0, T ; H)
\end{aligned}
$$

is continuous and the mapping $k \mapsto x_{k}$ is compact from $L^{2}(0, T ; H)$ to $L^{2}(0, T ; V)$, which is useful for physical applications for the equations related with forcing terms containing control part.

\section{Parabolic variational inequalities}

If $H$ is identified with its dual space we may write $V \subset H \subset V^{*}$ densely and the corresponding injections are continuous. The norm on $V, H$, and $V^{*}$ will be denoted by $\|\cdot\|$, $|\cdot|$, and $\|\cdot\|_{*}$, respectively. The duality pairing between the element $v_{1}$ of $V^{*}$ and the element $v_{2}$ of $V$ is denoted by $\left(v_{1}, v_{2}\right)$, which is the ordinary inner product in $H$ if $v_{1}, v_{2} \in H$. For the sake of simplicity, we may consider

$$
\|u\|_{*} \leq|u| \leq\|u\|, \quad u \in V
$$

For $l \in V^{*}$ we denote $(l, v)$ by the value $l(v)$ of $l$ at $v \in V$. The norm of $l$ as an element of $V^{*}$ is given by

$$
\|l\|_{*}=\sup _{v \in V} \frac{|(l, v)|}{\|v\|} .
$$

Therefore, we assume that $V$ has a stronger topology than $H$ and, for brevity, we may regard

$$
\|u\|_{*} \leq|u| \leq\|u\|, \quad \forall u \in V .
$$


Let $a(\cdot, \cdot)$ be a bounded sesquilinear form defined in $V \times V$ and satisfying Gårding's inequality,

$$
\operatorname{Re} a(u, u) \geq \omega_{1}\|u\|^{2}-\omega_{2}|u|^{2}
$$

where $\omega_{1}>0$ and $\omega_{2}$ is a real number.

Let $A$ be the operator associated with the sesquilinear form $a(\cdot, \cdot)$ :

$$
(A u, v)=a(u, v), \quad u, v \in V
$$

Then $A$ is a bounded linear operator from $V$ to $V^{*}$ by the Lax-Milgram theorem. The realization for the operator $A$ in $H$ which is the restriction of $A$ to

$$
D(A)=\{u \in V ; A u \in H\}
$$

can also be denoted by $A$. We also assume that there exists a constant $C_{1}$ such that

$$
\|u\| \leq C_{1}\|u\|_{D(A)}^{1 / 2}|u|^{1 / 2}
$$

for every $u \in D(A)$, where

$$
\|u\|_{D(A)}=\left(|A u|^{2}+|u|^{2}\right)^{1 / 2}
$$

is the graph norm of $D(A)$. Thus, in terms of the intermediate theory we may assume that

$$
(D(A), H)_{1 / 2,2}=V \text {, }
$$

where $(D(A), H)_{1 / 2,2}$ denotes the real interpolation space between $D(A)$ and $H$.

If $X$ is a Banach space, $L^{2}(0, T ; X)$ is the collection of all strongly measurable square integrable functions from $(0, T)$ into $X$ and $W^{1,2}(0, T ; X)$ is the set of all absolutely continuous functions on $[0, T]$ such that their derivative belongs to $L^{2}(0, T ; X) . C([0, T] ; X)$ will denote the set of all continuous functions from $[0, T]$ into $X$ with the supremum norm.

Lemma 2.1 Let $T>0$. Then

$$
H=\left\{x \in V^{*}: \int_{0}^{T}\left\|A e^{t A} x\right\|_{*}^{2} d t<\infty\right\} .
$$

Proof Put $u(t)=e^{t A} x$ for $x \in H$. From

$$
\begin{aligned}
\frac{1}{2} \frac{d}{d t}|u(t)|^{2} & =\operatorname{Re}(\dot{u}(t), u(t))=\operatorname{Re}(A u(t), u(t)) \\
& =-\operatorname{Re} a(u(t), u(t)) \leq-c_{0}\|u(t)\|^{2},
\end{aligned}
$$

it follows that

$$
\frac{1}{2} \frac{d}{d t}|u(t)|^{2}+c_{0}\|u(t)\|^{2} \leq 0 .
$$


Integrating over $t$ yields

$$
\frac{1}{2}|u(t)|^{2}+c_{0} \int_{0}^{t}\|u(s)\|^{2} d s \leq \frac{1}{2}|x|^{2} .
$$

Hence, we obtain

$$
\int_{0}^{T}\left\|A e^{t A} x\right\|_{*}^{2} d t \leq \int_{0}^{T}\|A\|_{B\left(V, V^{*}\right)}\|u(s)\|^{2} d s<\infty .
$$

Conversely, suppose that $x \in V^{*}$ and $\int_{0}^{T}\left\|A e^{t A} x\right\|_{*}^{2} d t<\infty$. Put $u(t)=e^{t A} x$. Then since $A$ is an isomorphism operator from $V$ to $V^{*}$ there exists a constant $c>0$ such that

$$
\int_{0}^{T}\|u(t)\|^{2} d t \leq c \int_{0}^{T}\|A u(t)\|_{*}^{2} d t=c \int_{0}^{T}\left\|A e^{t A} x\right\|_{*}^{2} d t .
$$

From the assumptions and $\dot{u}(t)=A e^{t A} x$ it follows that

$$
u \in L^{2}(0, T ; V) \cap W^{1,2}\left(0, T ; V^{*}\right) \subset C([0, T] ; H) .
$$

Therefore, $x=u(0) \in H$.

By Lemma 2.1, from Theorem 3.5.3 of Butzer and Berens [12], we can see that

$$
\left(V, V^{*}\right)_{1 / 2,2}=H \text {. }
$$

Using the regularity for the variational inequality of parabolic type (i.e., in case where $g \equiv 0)$ as seen in Section 4.3 of [8] we have the following result on (VE). We denote the closure in $H$ of the set $D(\phi)=\{u \in V: \phi(u)<\infty\}$ by $\overline{D(\phi)}$.

Proposition 2.1 (1) Let $k \in L^{2}\left(0, T ; V^{*}\right)$ and $\left(g^{0}, g^{1}\right) \in \overline{D(\phi)} \times L^{2}(-r, 0 ; V)$. Then (NDE) has a unique solution

$$
x \in L^{2}(0, T ; V) \cap W^{1,2}\left(0, T ; V^{*}\right) \cap C([0, T] ; H),
$$

which satisfies

$$
x^{\prime}(t)=(k(t)-A x(t)-\partial \phi(x(t)))^{0}
$$

and

$$
\|x\|_{L^{2} \cap W^{1,2} \cap C} \leq C_{2}\left(1+\left|g^{0}\right|+\left\|g^{1}\right\|_{L^{2}(-r, 0 ; V)}+\|k\|_{L^{2}\left(0, T ; V^{*}\right)}\right),
$$

where $C_{2}$ is some positive constant and $L^{2} \cap C=L^{2}(0, T ; V) \cap C([0, T] ; H)$.

(2) Let $A$ be symmetric and let us assume that there exists $h \in H$ such that for every $\epsilon>0$ and any $y \in D(\phi)$

$$
J_{\epsilon}(y+\epsilon h) \in D(\phi) \text { and } \phi\left(J_{\epsilon}(y+\epsilon h)\right) \leq \phi(y)
$$


where $J_{\epsilon}=(I+\epsilon A)^{-1}$. Then for $k \in L^{2}(0, T ; H)$ and $\left(g^{0}, g^{1}\right) \in \overline{D(\phi)} \cap V \times L^{2}(-r, 0 ; V)$, (NDE) has a unique solution,

$$
x \in L^{2}(0, T ; D(A)) \cap W^{1,2}(0, T ; H) \cap C([0, T] ; H),
$$

which satisfies

$$
\|x\|_{L^{2} \cap W^{1,2} \cap C} \leq C_{2}\left(1+\left|g^{0}\right|+\left\|g^{1}\right\|_{L^{2}(-r, 0 ; D(A))}+\|k\|_{L^{2}(0, T ; H)}\right) .
$$

Remark 2.1 In terms of Lemma 2.1, the inclusion

$$
L^{2}(0, T ; V) \cap W^{1,2}\left(0, T ; V^{*}\right) \subset C([0, T] ; H)
$$

is well known and is an easy consequence of the definition of real interpolation spaces by the trace method (see $[9,15])$.

\section{Regularity for nonlinear variational inequalities}

Let $\mathcal{L}$ and $\mathcal{B}$ be the Lebesgue $\sigma$-field on $[0, \infty)$ and the Borel $\sigma$-field on $[-r, 0]$, respectively. Let $\mu$ be a Borel measure on $[-r, 0]$ and $h:[0, \infty) \times[-r, 0] \times V \times H \rightarrow H$ be a nonlinear mapping satisfying the following:

(G1) for any $x, y \in V$ the mapping $h(\cdot, \cdot, x, y)$ is strongly $\mathcal{L} \times \mathcal{B}$-measurable;

(G2) there exist positive constants $L_{i}(i=0,1,2)$ such that

$$
\begin{aligned}
& |h(t, s, x, y)-h(t, s, \hat{x}, \hat{y})| \leq L_{1}\|x-\hat{x}\|+L_{2}|y-\hat{y}|, \\
& |h(t, s, 0,0)| \leq L_{0}
\end{aligned}
$$$$
\text { for all }(t, s) \in[0, \infty) \times[-r, 0] \text { and } x, \hat{x}, y, \hat{y} \in V \text {. }
$$

Remark 3.1 The above operator $h$ is the semilinear case of the nonlinear part of quasilinear equations considered by Yong and Pan [11].

For any $x \in L^{2}(-r, T ; V), T>0$ we set

$$
G(t, x)=\int_{-r}^{0} h(t, s, x(t), x(t+s)) \mu(d s) .
$$

Here as in [11] we consider the Borel measurable corrections of $x(\cdot)$.

Lemma 3.1 Let $x \in L^{2}(-r, T ; V), T>0$. Then the nonlinear term $G(\cdot, x)$ defined by $(3.1)$ belongs to $L^{2}(0, T ; H)$ and

$$
\|G(\cdot, x)\|_{L^{2}(0, T ; H)} \leq \mu([-r, 0])\left\{L_{0} \sqrt{T}+\left(L_{1}+L_{2}\right)\|x\|_{L^{2}(0, T ; V)}+L_{2}\|x\|_{L^{2}(-r, 0 ; V)}\right\} .
$$

Moreover, if $x_{1}, x_{2} \in L^{2}(-r, T ; V)$, then

$$
\begin{aligned}
& \left\|G\left(\cdot, x_{1}\right)-G\left(\cdot, x_{2}\right)\right\|_{L^{2}(0, T ; H)} \\
& \quad \leq \mu([-r, 0])\left\{\left(L_{1}+L_{2}\right)\left\|x_{1}-x_{2}\right\|_{L^{2}(0, T ; V)}+L_{2}\left\|x_{1}-x_{2}\right\|_{L^{2}(-r, 0 ; V)}\right\} .
\end{aligned}
$$


Proof From (G1) and (G2) it is easily seen that

$$
\begin{aligned}
& \|G(\cdot, x)\|_{L^{2}(0, T ; H)} \\
& \quad \leq \mu([-r, 0])\left\{L_{0} \sqrt{T}+L_{1}\|x\|_{L^{2}(0, T ; V)}+L_{2}\|x\|_{L^{2}(-r, T ; V)}\right\} \\
& \quad \leq \mu([-r, 0])\left\{L_{0} \sqrt{T}+\left(L_{1}+L_{2}\right)\|x\|_{L^{2}(0, T ; V)}+L_{2}\|x\|_{L^{2}(-r, 0 ; V)}\right\} .
\end{aligned}
$$

The proof of (3.3) is similar.

We denote the closure in $H$ of the set $D(\phi)=\{u \in V: \phi(u)<\infty\}$ by $\overline{D(\phi)}$. In what follows this paper, we assume that $\overline{D(\phi)}=H$ for the sake of simplicity. First, we are going to give the following result on a local solvability of (NDE). The following lemma is due to Brézis [4, Lemma A.5].

Lemma 3.2 Let $m \in L^{1}(0, T ; \mathbb{R})$ satisfying $m(t) \geq 0$ for all $t \in(0, T)$ and $a \geq 0$ be a constant. Let $b$ be a continuous function on $[0, T] \subset \mathbb{R}$ satisfying the following inequalities:

$$
\frac{1}{2} b^{2}(t) \leq \frac{1}{2} a^{2}+\int_{0}^{t} m(s) b(s) d s, \quad t \in[0, T]
$$

Then

$$
|b(t)| \leq a+\int_{0}^{t} m(s) d s, \quad t \in[0, T] .
$$

Let $x \in L^{2}(0, T ; V) \cap C([0, T] ; H)$. Then invoking Proposition 2.1 , we see that the problem

$$
\left\{\begin{array}{l}
y^{\prime}(t)+A y(t)+\partial \phi(y(t)) \ni G(t, x)+k(t), \quad 0<t \leq T, \\
y(0)=g^{0}, \quad y(s)=g^{1}(s), \quad-r \leq s \leq 0,
\end{array}\right.
$$

has a unique solution $y \in L^{2}(0, T ; V) \cap W^{1,2}\left(0, T ; V^{*}\right) \cap C([0, T] ; H)$.

Lemma 3.3 Let $y_{1}, y_{2}$ be the solutions of (3.4) with $x$ replaced by $x_{1}, x_{2} \in L^{2}(0, T ; V)$, respectively. Then the following inequalities hold:

$$
\begin{gathered}
\frac{1}{2}\left|y_{1}(t)-y_{2}(t)\right|^{2}+\omega_{1} \int_{0}^{t}\left\|y_{1}(s)-y_{2}(s)\right\|^{2} d s \\
\leq \int_{0}^{t} e^{2 \omega_{2}(t-s)} H(s)\left|y_{1}(s)-y_{2}(s)\right| d s
\end{gathered}
$$

and

$$
\left|y_{1}(t)-y_{2}(t)\right| \leq \int_{0}^{t} e^{\omega_{2}(t-s)} H(s) d s
$$

where

$$
H(t)=\mu([-r, 0]) L_{1}\left\|x_{1}(t)-x_{2}(t)\right\|+\mu([-r, 0]) L_{2}\left\|x_{1}-x_{2}\right\|_{C([0, t] ; H)} .
$$


Proof For $i=1,2$, we consider the following equation:

$$
\left\{\begin{array}{l}
y_{i}^{\prime}(t)+A y_{i}(t)+\partial \phi\left(y_{i}(t)\right) \ni G\left(t, x_{i}\right)+k(t), \quad 0<t \leq T, \\
y_{i}(0)=g^{0}, \quad y_{i}(s)=g^{1}(s), \quad-r \leq s \leq 0 .
\end{array}\right.
$$

From (3.7) it follows that

$$
\begin{aligned}
& \frac{d}{d t}\left(y_{1}(t)-y_{2}(t)\right)+A\left(y_{1}(t)-y_{2}(t)\right)+\partial \phi\left(y_{1}(t)\right)-\partial \phi\left(y_{2}(t)\right) \\
& \quad \ni G\left(t, x_{1}\right)-G\left(t, x_{2}\right), \quad t>0 .
\end{aligned}
$$

Multiplying on both sides of $y_{1}(t)-y_{2}(t)$ and using the monotonicity of $\partial \phi$, we get

$$
\begin{aligned}
& \frac{1}{2} \frac{d}{d t}\left|y_{1}(t)-y_{2}(t)\right|^{2}+a\left(y_{1}(t)-y_{2}(t), y_{1}(t)-y_{2}(t)\right) \\
& \quad \leq\left(G\left(t, x_{1}\right)-G\left(t, x_{2}\right), y_{1}(t)-y_{2}(t)\right) .
\end{aligned}
$$

Noting that since $x_{1}(s)-x_{2}(s)=0$ for $s \in[-r, 0)$,

$$
\left|G\left(t, x_{1}\right)-G\left(t, x_{2}\right)\right| \leq \mu([-r, 0]) L_{1}\left\|x_{1}(t)-x_{2}(t)\right\|+\mu([-r, 0]) L_{2}\left\|x_{1}-x_{2}\right\|_{C([0, t] ; H)},
$$

and putting

$$
H(t)=\mu([-r, 0]) L_{1}\left\|x_{1}(t)-x_{2}(t)\right\|+\mu([-r, 0]) L_{2}\left\|x_{1}-x_{2}\right\|_{C([0, t] ; H)},
$$

by (2.1) and (2.2), we have

$$
\begin{aligned}
& \frac{1}{2} \frac{d}{d t}\left|y_{1}(t)-y_{2}(t)\right|^{2}+\omega_{1}\left\|y_{1}(t)-y_{2}(t)\right\|^{2} \\
& \quad \leq \omega_{2}\left|y_{1}(t)-y_{2}(t)\right|^{2}+H(t)\left|y_{1}(t)-y_{2}(t)\right| .
\end{aligned}
$$

Hence, integrating $(3.8)$ over $(0, t)$, this yields

$$
\begin{aligned}
& \frac{1}{2}\left|y_{1}(t)-y_{2}(t)\right|^{2}+\omega_{1} \int_{0}^{t}\left\|y_{1}(s)-y_{2}(s)\right\|^{2} d s \\
& \quad \leq \omega_{2} \int_{0}^{t}\left|y_{1}(s)-y_{2}(s)\right|^{2} d s+\int_{0}^{t} H(s)\left|y_{1}(s)-y_{2}(s)\right| d s .
\end{aligned}
$$

From (3.9) it follows that

$$
\begin{aligned}
& \frac{d}{d t}\left\{e^{-2 \omega_{2} t} \int_{0}^{t}\left|y_{1}(s)-y_{2}(s)\right|^{2} d s\right\} \\
& \quad=2 e^{-2 \omega_{2} t}\left\{\frac{1}{2}\left|y_{1}(t)-y_{2}(t)\right|^{2}-\omega_{2} \int_{0}^{t}\left|y_{1}(s)-y_{2}(s)\right|^{2} d s\right\} \\
& \quad \leq 2 e^{-2 \omega_{2} t} \int_{0}^{t} H(s)\left|y_{1}(s)-y_{2}(s)\right| d s .
\end{aligned}
$$


Integrating (3.10) over $(0, t)$ we have

$$
\begin{aligned}
& e^{-2 \omega_{2} t} \int_{0}^{t}\left|y_{1}(s)-y_{2}(s)\right|^{2} d s \\
& \leq 2 \int_{0}^{t} e^{-2 \omega_{2} \tau} \int_{0}^{\tau} H(s)\left|y_{1}(s)-y_{2}(s)\right| d s d \tau \\
& =\frac{1}{\omega_{2}} \int_{0}^{t}\left(e^{-2 \omega_{2} s}-e^{-2 \omega_{2} t}\right) H(s)\left|y_{1}(s)-y_{2}(s)\right| d s,
\end{aligned}
$$

thus, we get

$$
\omega_{2} \int_{0}^{t}\left|y_{1}(s)-y_{2}(s)\right|^{2} d s \leq \int_{0}^{t}\left(e^{2 \omega_{2}(t-s)}-1\right) H(s)\left|y_{1}(s)-y_{2}(s)\right| d s .
$$

From (3.9) and (3.11), (3.5) holds, which implies

$$
\begin{aligned}
& \frac{1}{2}\left(e^{-2 \omega_{2} t}\left|y_{1}(t)-y_{2}(t)\right|\right)^{2}+\omega_{1} e^{-2 \omega_{2} t} \int_{0}^{t}\left\|y_{1}(s)-y_{2}(s)\right\|^{2} d s \\
& \quad \leq \int_{0}^{t} e^{-\omega_{2} s} H(s) e^{-\omega_{2} s}\left|y_{1}(s)-y_{2}(s)\right| d s .
\end{aligned}
$$

By using Lemma 3.1, we obtain (3.6), as claimed.

Theorem 3.1 Let the assumptions (G1) and (G2) be satisfied. Assume that $k \in L^{2}\left(0, T ; V^{*}\right)$ and $\left(g^{0}, g^{1}\right) \in H \times L^{2}(-r, 0 ; V)$. Then there exists a time $T_{0}>0$ such that the functional differential equation (NDE) admits a unique solution $x$ in $L^{2}\left(-r, T_{0} ; V\right) \cap C\left(\left[0, T_{0}\right] ; V^{*}\right)$.

Proof Let us fix $T_{0}>0$ such that

$$
\frac{M\left(e^{2 \omega_{2} T_{0}}-1\right)}{4 \omega_{2} \min \left\{1 / 2, \omega_{2}\right\}}<1
$$

where

$$
M \equiv\left(L_{1} \mu([-r, 0])\right)^{2}+2 L_{1} L_{2} \mu([-r, 0])^{2}+\left(L_{2} \mu([-r, 0])\right)^{2} .
$$

We are going to show that $x \mapsto y$ is strictly contractive from $L^{2}\left(0, T_{0} ; V\right) \cap C\left(\left[0, T_{0}\right] ; H\right)$ to itself if the condition (3.12) is satisfied. The norm in $L^{2}\left(0, T_{0} ; V\right) \cap C\left(\left[0, T_{0}\right] ; H\right)$ is given by

$$
\|\cdot\|_{L^{2}\left(0, T_{0} ; V\right) \cap C\left(\left[0, T_{0}\right] ; H\right)}=\max \left\{\|\cdot\|_{L^{2}\left(0, T_{0} ; V\right)},\|\cdot\|_{C\left(\left[0, T_{0}\right] ; H\right)}\right\} .
$$

Let $y_{1}, y_{2}$ be the solutions of (3.7) with $x$ replaced by $x_{1}, x_{2} \in L^{2}\left(0, T_{0} ; V\right)$, respectively. From (3.5) and (3.6) it follows that

$$
\begin{gathered}
\frac{1}{2}\left|y_{1}(t)-y_{2}(t)\right|^{2}+\omega_{1} \int_{0}^{t}\left\|y_{1}(s)-y_{2}(s)\right\|^{2} d s \\
\leq \int_{0}^{t} e^{2 \omega_{2}(t-s)} H(s) \int_{0}^{s} e^{\omega_{2}(s-\tau)} H(\tau) d \tau d s
\end{gathered}
$$




$$
\begin{aligned}
& =e^{2 \omega_{2} t} \int_{0}^{t} e^{-\omega_{2} s} H(s) \int_{0}^{s} e^{-\omega_{2} \tau} H(\tau) d \tau d s \\
& =e^{2 \omega_{2} t} \int_{0}^{t} \frac{1}{2} \frac{d}{d s}\left\{\int_{0}^{s} e^{-\omega_{2} \tau} H(\tau) d \tau\right\}^{2} d s=\frac{1}{2} e^{2 \omega_{2} t}\left\{\int_{0}^{t} e^{-\omega_{2} \tau} H(\tau) d \tau\right\}^{2} \\
& \leq \frac{1}{2} e^{2 \omega_{2} t} \int_{0}^{t} e^{-2 \omega_{2} \tau} d \tau \int_{0}^{t} H(\tau)^{2} d \tau=\frac{1}{2} e^{2 \omega_{2} t} \frac{1-e^{-2 \omega_{2} t}}{2 \omega_{2}} \int_{0}^{t} H(\tau)^{2} d \tau \\
& =\frac{1}{4 \omega_{2}}\left(e^{2 \omega_{2} t}-1\right) \int_{0}^{t} H(s)^{2} d s
\end{aligned}
$$

Here, since

$$
\begin{aligned}
\int_{0}^{t} H(s)^{2} d s= & \int_{0}^{t}\left\{\left(L_{1} \mu([-r, 0])\right)^{2}\left\|x_{1}(s)-x_{2}(s)\right\|^{2}\right. \\
& +2 L_{1} L_{2} \mu([-r, 0])^{2}\left\|x_{1}(s)-x_{2}(s)\right\| \cdot\left\|x_{1}-x_{2}\right\|_{C([0, s] ; H)} \\
& \left.+\left(L_{2} \mu([-r, 0])\right)^{2}\left\|x_{1}-x_{2}\right\|_{C([0, s] ; H)}^{2}\right\} d s \\
\leq & \left\{\left(L_{1} \mu([-r, 0])\right)^{2}+2 L_{1} L_{2} \mu([-r, 0])^{2}\right. \\
& \left.+\left(L_{2} \mu([-r, 0])\right)^{2}\right\}\left\|x_{1}-x_{2}\right\|_{L^{2}(0, t ; V) \cap C([0, t] ; H)}^{2},
\end{aligned}
$$

taking

$$
M \equiv\left(L_{1} \mu([-r, 0])\right)^{2}+2 L_{1} L_{2} \mu([-r, 0])^{2}+\left(L_{2} \mu([-r, 0])\right)^{2}
$$

from (3.13) it follows that

$$
\left\|y_{1}-y_{2}\right\|_{L^{2}\left(0, T_{0} ; V\right) \cap C\left(\left[0, T_{0}\right] ; H\right)}^{2} \leq \frac{M\left(e^{2 \omega_{2} T_{0}}-1\right)}{4 \omega_{2} \min \left\{1 / 2, \omega_{2}\right\}}\left\|x_{1}-x_{2}\right\|_{L^{2}\left(0, T_{0} ; V\right) \cap C\left(\left[0, T_{0}\right] ; H\right)}^{2} .
$$

Starting from initial value $x_{0}(t)=g^{0}, x_{0}(s)=g^{1}(s)$ for $-r \leq s \leq 0$, consider a sequence $\left\{x_{n}(\cdot)\right\}$ satisfying

$$
\left\{\begin{array}{l}
x_{n+1}^{\prime}(t)+A x_{n+1}(t)+\partial \phi\left(x_{n+1}(t)\right) \ni G\left(t, x_{n}\right)+k(t), \quad 0<t \leq T \\
x_{n+1}(0)=g^{0}, \quad x_{n+1}^{\prime}(s)=g^{1}(s), \quad-r \leq s \leq 0 .
\end{array}\right.
$$

Then from (3.14) it follows that

$$
\left\|x_{n+1}-x_{n}\right\|_{L^{2}\left(0, T_{0} ; V\right) \cap C\left(\left[0, T_{0}\right] ; H\right)}^{2} \leq \frac{M\left(e^{2 \omega_{2} T_{0}}-1\right)}{4 \omega_{2} \min \left\{1 / 2, \omega_{2}\right\}}\left\|x_{n}-x_{n-1}\right\|_{L^{2}\left(0, T_{0} ; V\right) \cap C\left(\left[0, T_{0}\right] ; H\right)}^{2}
$$

So by virtue of the condition (3.12) the contraction principle shows that there exists $x(\cdot) \in$ $L^{2}\left(0, T_{0} ; V\right) \cap C\left(\left[0, T_{0}\right] ; H\right)$ such that

$$
x_{n}(\cdot) \rightarrow x(\cdot) \quad \text { in } L^{2}\left(0, T_{0} ; V\right) \cap C\left(\left[0, T_{0}\right] ; H\right) .
$$

Now we give a norm estimation of the solution of (NDE) and establish the global existence of solutions with the aid of norm estimations. 
Theorem 3.2 Let the assumptions (G1) and (G2) be satisfied. Then for any $\left(g^{0}, g^{1}\right) \in H \times$ $L^{2}(-r, 0 ; V)(T>0)$ and $k \in L^{2}\left(0, T ; V^{*}\right)$, the solution $x$ of $(\mathrm{NDE})$ exists and is unique in $\mathcal{W}_{1}(T) \equiv L^{2}(-r, T ; V) \cap W^{1,2}\left(0, T ; V^{*}\right)$, and there exists a constant $C_{2}$ depending on $T$ such that

$$
\|x\|_{\mathcal{W}_{1}(T)} \leq C_{2}\left(1+\left|g^{0}\right|+\left\|g^{1}\right\|_{L^{2}(-r, 0 ; V)}+\|k\|_{L^{2}\left(0, T ; V^{*}\right)}\right)
$$

Proof Let $y$ be the solution of

$$
\left\{\begin{array}{l}
\frac{d y(t)}{d t}+A y(t)+\partial \phi(y(t)) \ni k(t), \quad 0<t \leq T_{0} \\
y(0)=g^{0}
\end{array}\right.
$$

Then, since

$$
\frac{d}{d t}(x(t)-y(t))+A(x(t)-y(t))+\partial \phi(x(t))-\partial \phi(y(t)) \ni G(t, x)
$$

by multiplying by $x(t)-y(t)$ and using the monotonicity of $\partial \phi,(2.1)$, and (2.2), we obtain

$$
\begin{aligned}
& \frac{1}{2} \frac{d}{d t}|x(t)-y(t)|^{2}+\omega_{1}|| x(t)-y(t) \|^{2} \\
& \quad \leq \omega_{2}|x(t)-y(t)|^{2}+|G(t, x)| \cdot|x(t)-y(t)| .
\end{aligned}
$$

By integrating on $(3.16)$ over $(0, t)$ we have

$$
\begin{aligned}
& \frac{1}{2}|x(t)-y(t)|^{2}+\omega_{1} \int_{0}^{t}\|x(s)-y(s)\|^{2} d s \\
& \quad \leq \omega_{2} \int_{0}^{t}|x(s)-y(s)|^{2} d s+\int_{0}^{t}|G(s, x)| \cdot|x(s)-y(s)| d s .
\end{aligned}
$$

By a procedure similar to (3.13) regarding

$$
H(t)=\mu([-r, 0]) L_{1}\|x(t)\|+\mu([-r, 0]) L_{2}\|x\|_{C([0, t] ; H)},
$$

we have

$$
\|x-y\|_{L^{2}\left(0, T_{0} ; V\right) \cap C\left(\left[0, T_{0}\right] ; H\right)}^{2} \leq \frac{M\left(e^{2 \omega_{2} T_{0}}-1\right)}{4 \omega_{2} \min \left\{1 / 2, \omega_{2}\right\}}\|x\|_{L^{2}\left(0, T_{0} ; V\right) \cap C\left(\left[0, T_{0}\right] ; H\right)}^{2} .
$$

Put

$$
N=\frac{M\left(e^{2 \omega_{2} T_{0}}-1\right)}{4 \omega_{2} \min \left\{1 / 2, \omega_{2}\right\}} .
$$

Then we have

$$
\|x-y\|_{L^{2}\left(0, T_{0} ; V\right) \cap C\left(\left[0, T_{0}\right] ; H\right)} \leq N^{1 / 2}\|x\|_{L^{2}\left(0, T_{0} ; V\right) \cap C\left(\left[0, T_{0}\right] ; H\right)}
$$


and hence, from (2.4) in Proposition 2.1, we have

$$
\begin{aligned}
& \|x\|_{L^{2}\left(0, T_{0} ; V\right) \cap C\left(\left[0, T_{0}\right] ; H\right)} \\
& \quad \leq \frac{1}{1-N^{1 / 2}}\|y\|_{L^{2}\left(0, T_{0} ; V\right) \cap C\left(\left[0, T_{0}\right] ; H\right)} \\
& \quad \leq \frac{C_{2}}{1-N^{1 / 2}}\left(1+\left|g^{0}\right|+\left\|g^{1}\right\|_{L^{2}\left(0, T_{0} ; V\right)}+\|k\|_{L^{2}\left(0, T_{0} ; V^{*}\right)}\right) \\
& \quad \leq C_{3}\left(1+\left|g^{0}\right|+\left\|g^{1}\right\|_{L^{2}\left(0, T_{0} ; V\right)}+\|k\|_{L^{2}\left(0, T_{0} ; V^{*}\right)}\right)
\end{aligned}
$$

for some positive constant $C_{3}$. Acting on both side of (NDE) by $x^{\prime}(t)$ and by using

$$
\frac{d}{d t} \phi(x(t))=\left(g(t), \frac{d}{d t} x(t)\right), \quad \text { a.e. } 0<t
$$

for all $g(t) \in \partial \phi(x(t))$, we have

$$
\begin{aligned}
& \int_{0}^{t}\left|x_{n}^{\prime}(s)\right|^{2} d s+\frac{1}{2}\left(A x_{n}(t), x_{n}(t)\right)+\phi\left(x_{n}(t)\right) \\
& \quad \leq \frac{1}{2}\left(A x_{0}, x_{0}\right)+\phi\left(x_{0}\right)+\int_{0}^{t}\left|G\left(s, x_{n}(s)\right)+k(s)\right|\left|x_{n}^{\prime}(s)\right| d s,
\end{aligned}
$$

thus, we obtain the norm estimate of $x$ in $W^{1,2}(0, T ; H)$ satisfying (3.15). Since the condition (3.12) is independent of initial values we can derive from (3.19) that $\phi\left(x\left(n T_{0}\right)\right)<\infty$, and the solution of (NDE) can be extended to the internal $\left[0, n T_{0}\right]$ for the natural number $n$, i.e., for the initial $x\left(n T_{0}\right)$ in the interval $\left[n T_{0},(n+1) T_{0}\right]$, an analogous estimate (3.18) holds for the solution in $\left[0,(n+1) T_{0}\right]$. Furthermore, the estimate $(3.15)$ is easily obtained from (3.18) and (3.19).

Theorem 3.3 Suppose that the assumptions (G1) and (G2) are satisfied. Let $A$ be symmetric and let us assume that there exists $h \in H$ such that for every $\epsilon>0$ and any $y \in D(\phi)$

$$
\begin{gathered}
J_{\epsilon}(y+\epsilon h) \in D(\phi) \quad \text { and } \quad \phi\left(J_{\epsilon}(y+\epsilon h)\right) \leq \phi(y) . \\
\text { If } k \in L^{2}(0, T ; H) \text { and }\left(g^{0}, g^{1}\right) \in V \times L^{2}(-r, 0 ; D(A)) \text {, then } \\
x \in \mathcal{W}_{0}(T) \equiv L^{2}(-r, T ; D(A)) \cap W^{1,2}(0, T ; H),
\end{gathered}
$$

and the mapping $\left(g^{0}, g^{1}, k\right) \mapsto u \in \mathcal{W}_{0}(T)$ is continuous.

Proof It is easy to show that if $\left(g^{0}, g^{1}\right) \in V \times L^{2}(-r, 0 ; D(A))$ and $k \in L^{2}(0, T ; H)$, then from Proposition 2.1 it follows that $u$ belongs to $\mathcal{W}_{0}(T)$. Let $\left(g_{i}^{0}, g_{i}^{1}, k_{i}\right) \in V \times L^{2}(-r, 0 ; D(A)) \times$ $L^{2}(0, T ; H)$, and $u_{i}$ be the solution of (NDE) with $\left(g_{i}^{0}, g_{i}^{1}, k_{i}\right)$ in place of $\left(g^{0}, g^{1}, k\right)$ for $i=1,2$. Then in view of Proposition 2.1 and Lemma 2.1 we have

$$
\begin{aligned}
\left\|x_{1}-x_{2}\right\| \mathcal{W}_{0}(T) \leq & C_{2}\left\{\left\|g_{1}^{0}-g_{2}^{0}\right\|+\left\|g_{1}^{1}-g_{2}^{1}\right\|_{L^{2}(-r, 0 ; D(A))}\right. \\
& \left.+\left\|G\left(\cdot, x_{1}\right)-G\left(\cdot, x_{2}\right)\right\|_{L^{2}(0, T ; H)}+\left\|k_{1}-k_{2}\right\|_{L^{2}(0, T ; H)}\right\}
\end{aligned}
$$




$$
\begin{aligned}
\leq & C_{2}\left[\left\|g_{1}^{0}-g_{2}^{0}\right\|+\left\|g_{1}^{1}-g_{2}^{1}\right\|_{L^{2}(-r, 0 ; D(A))}+\left\|k_{1}-k_{2}\right\|_{L^{2}(0, T ; H)}\right. \\
& +\mu([-r, 0])\left\{( L _ { 1 } + L _ { 2 } ) \left(\left\|x_{1}-x_{2}\right\|_{L^{2}(0, T ; V)}\right.\right. \\
& \left.\left.\left.+L_{2}\left\|g_{1}^{1}-g_{2}^{1}\right\|_{L^{2}(-r, 0 ; V)}\right)\right\}\right] .
\end{aligned}
$$

Since

$$
x_{1}(t)-x_{2}(t)=g_{1}^{0}-g_{2}^{0}+\int_{0}^{t}\left(\dot{x}_{1}(s)-\dot{x}_{2}(s)\right) d s,
$$

we get

$$
\left\|x_{1}-x_{2}\right\|_{L^{2}(0, T ; H)} \leq \sqrt{T}\left|g_{1}^{0}-g_{2}^{0}\right|+\frac{T}{\sqrt{2}}\left\|x_{1}-x_{2}\right\|_{W^{1,2}(0, T ; H)} .
$$

Hence, arguing as in (2.3) we get

$$
\begin{aligned}
\left\|x_{1}-x_{2}\right\|_{L^{2}(0, T ; V)} \leq & C_{1}\left\|x_{1}-x_{2}\right\|_{L^{2}(0, T ; D(A))}^{1 / 2}\left\|x_{1}-x_{2}\right\|_{L^{2}(0, T ; H)}^{1 / 2} \\
\leq & C_{1}\left\|x_{1}-x_{2}\right\|_{L^{2}(0, T ; D(A))}^{1 / 2} \\
& \times\left\{T^{1 / 4}\left|g_{1}^{0}-g_{2}^{0}\right|^{1 / 2}+\left(\frac{T}{\sqrt{2}}\right)^{1 / 2}\left\|x_{1}-x_{2}\right\|_{W^{1,2}(0, T ; H)}^{1 / 2}\right\} \\
\leq & C_{1} T^{1 / 4}\left|g_{1}^{0}-g_{2}^{0}\right|^{1 / 2}\left\|x_{1}-x_{2}\right\|_{L^{2}(0, T ; D(A))}^{1 / 2}+C_{1}\left(\frac{T}{\sqrt{2}}\right)^{1 / 2}\left\|x_{1}-x_{2}\right\| \mathcal{W}_{0}(T) \\
\leq & 2^{-7 / 4} C_{1}\left|g_{1}^{0}-g_{2}^{0}\right|+2 C_{1}\left(\frac{T}{\sqrt{2}}\right)^{1 / 2}\left\|x_{1}-x_{2}\right\|_{\mathcal{W}_{0}(T)} .
\end{aligned}
$$

Combining (3.20) and (3.21) we obtain

$$
\begin{aligned}
\left\|x_{1}-x_{2}\right\| \mathcal{W}_{0}(T) \leq & C_{2}\left\{\left\|g_{1}^{0}-g_{2}^{0}\right\|+\left\|g_{1}^{1}-g_{2}^{1}\right\|_{L^{2}(-r, 0 ; D(A))}\right. \\
& \left.+\left\|k_{1}-k_{2}\right\|_{L^{2}(0, T ; H)}+\mu([-r, 0]) L_{2}\left\|g_{1}^{1}-g_{2}^{1}\right\|_{L^{2}(-r, 0 ; V)}\right\} \\
& +2^{-7 / 4} C_{1} C_{2} \mu([-r, 0])\left(L_{1}+L_{2}\right)\left|g_{1}^{0}-g_{2}^{0}\right|+2 C_{1} C_{2}\left(\frac{T}{\sqrt{2}}\right)^{1 / 2} \\
& \times \mu([-r, 0])\left(L_{1}+L_{2}\right)\left\|x_{1}-x_{2}\right\| \mathcal{W}_{0}(T) .
\end{aligned}
$$

Suppose that $\left(g_{n}^{0}, g_{n}^{1}, k_{n}\right) \rightarrow\left(g^{0}, g^{1}, k\right)$ in $X \times L^{2}(-r, 0 ; D(A)) \times L^{2}(0, T ; H)$, and let $x_{n}$ and $x$ be the solutions (SLE) with $\left(g_{n}^{0}, g_{n}^{1}, k_{n}\right)$ and $\left(g^{0}, g^{1}, k\right)$, respectively. Let $0<T_{1} \leq T$ be such that

$$
2 C_{1} C_{2}\left(T_{1} / \sqrt{2}\right)^{1 / 2}\left(L_{1}+L_{2} L_{3} T_{1} / \sqrt{2}\right)<1 .
$$

Then by virtue of (3.22) with $T$ replaced by $T_{1}$ we see that $u_{n} \rightarrow u$ in $\mathcal{W}_{0}\left(T_{1}\right)$. This implies that $\left(x_{n}\left(T_{1}\right),\left(x_{n}\right)_{T_{1}}\right) \mapsto\left(x\left(T_{1}\right), x_{T_{1}}\right)$ in $V \times L^{2}(-r, 0 ; D(A))$. Hence the same argument shows that $u_{n} \rightarrow u$ in

$$
L^{2}\left(T_{1}, \min \left\{2 T_{1}, T\right\} ; D(A)\right) \cap W^{1,2}\left(T_{1}, \min \left\{2 T_{1}, T\right\} ; H\right) .
$$

Repeating this process we conclude that $u_{n} \rightarrow u$ in $\mathcal{W}_{0}(T)$. 
Theorem 3.4 For $k \in L^{2}(0, T ; H)$ let $x_{k}$ be the solution of $(\mathrm{NDE})$. Let us assume the natural assumption that the embedding $D(A) \subset V$ is compact. Then the mapping $k \mapsto x_{k}$ is compact from $L^{2}(0, T ; H)$ to $L^{2}(0, T ; V)$.

Proof If $k \in L^{2}(0, T ; H)$, noting $\|k\|_{L^{2}\left(0, T ; V^{*}\right)} \leq\|k\|_{L^{2}(0, T ; H)}$, then in view of Theorem 3.2

$$
\left\|x_{k}\right\|_{\mathcal{W}_{1}(T)} \leq C_{2}\left(1+\left|g^{0}\right|+\left\|g^{1}\right\|_{L^{2}(-r, 0 ; V)}+\|k\|_{L^{2}(0, T ; H)}\right)
$$

Since $x_{k} \in L^{2}(0, T ; V), G\left(\cdot, x_{k}\right) \in L^{2}(0, T ; H)$. Consequently $x_{k} \in L^{2}(0, T ; D(A)) \cap W^{1,2}(0, T$; $H$ ) and with aid of Proposition 2.1, Lemma 3.1, and (3.23),

$$
\begin{aligned}
\left\|x_{k}\right\|_{L^{2}(0, T ; D(A)) \cap W^{1,2}(0, T ; V)} & \\
\leq & C_{2}\left(\left\|g^{0}\right\|+\left\|g^{1}\right\|_{L^{2}(-r, 0 ; D(A))}+\left\|G\left(\cdot, x_{k}\right)+k\right\|_{L^{2}(0, T ; H)}\right) \\
\leq & C_{2}\left\{\left\|g^{0}\right\|+\left\|g^{1}\right\|_{L^{2}(-r, 0 ; D(A))}+\mu([-r, 0]) L_{0} \sqrt{T}\right. \\
& \left.+\mu([-r, 0])\left(L_{1}+L_{2}\right)\|x\|_{L^{2}(0, T ; V)}+\mu([-r, 0]) L_{2}\left\|g^{1}\right\|_{L^{2}(-r, 0 ; V)}+\|k\|_{L^{2}(0, T ; H)}\right\} \\
\leq & C_{2}\left[\left\|g^{0}\right\|+\left\|g^{1}\right\|_{L^{2}(-r, 0 ; D(A))}+\mu([-r, 0]) L_{0} \sqrt{T}\right. \\
& +\mu([-r, 0])\left(L_{1}+L_{2}\right) C_{2}\left\{1+\left|g^{0}\right|+\left\|g^{1}\right\|_{L^{2}(-r, 0 ; V)}+\|k\|_{L^{2}(0, T ; H)}\right\} \\
& \left.+\mu([-r, 0]) L_{2}\left\|g^{1}\right\|_{L^{2}(-r, 0 ; V)}+\|k\|_{L^{2}(0, T ; H)}\right] .
\end{aligned}
$$

Hence if $k$ is bounded in $L^{2}(0, T ; H)$, then so is $x_{k}$ in $L^{2}(0, T ; D(A)) \cap W^{1,2}(0, T ; H)$. Since $D(A)$ is compactly embedded in $V$ by assumption, the embedding

$$
L^{2}(0, T ; D(A)) \cap W^{1,2}(0, T ; H) \subset L^{2}(0, T ; V)
$$

is compact in view of Theorem 2 of Aubin [16].

Remark 3.2 Since the operator $A: D(A) \subset H \rightarrow H$ is an unbounded operator, we will make use of the hypothesis (G2). If $A$ is a bounded operator from $H$ into itself, we may assume that $h:[0, \infty) \times[-r, 0] \times V \times H \rightarrow H$ is a nonlinear mapping satisfying the following: there exist positive constants $L_{i}(i=1,2)$ such that

$$
|h(t, s, x, y)-h(t, s, \hat{x}, \hat{y})| \leq L_{1}|x-\hat{x}|+L_{2}|y-\hat{y}|
$$

for all $(t, s) \in[0, \infty) \times[-r, 0]$ and $x, \hat{x}, y, \hat{y} \in V$, then our results can be obtained directly.

\section{Competing interests}

The authors declare that they have no competing interests.

\section{Authors' contributions}

$\mathrm{H}-\mathrm{HR}$ drafted the manuscript and corrected the main results, J-MJ carried out the main proof of this paper and participated in its design and coordination. Both authors read and approved the final manuscript.

\section{Acknowledgements}

This research was supported by Basic Science Research Program through the National research Foundation of Korea (NRF) funded by the Ministry of Education, Science and Technology (2014045161). 


\section{References}

1. Lions, JL: Quelques méthodes de résolution des problèmes aux limites non linéaires. Gauthier-Villars, Paris (1969)

2. Lions, JL, Magenes, E: Non-Homogeneous Boundary value Problems and Applications. Springer, Berlin (1972)

3. Brézis, H: Problèmes unilatéraux. J. Math. Pures Appl. 51, 1-168 (1972)

4. Brézis, H: Opérateurs Maximaux Monotones et Semigroupes de Contractions dans les Espaces de Hilbert. North-Holland, Amsterdam (1973)

5. Friedman, A: Variational Principle and Free-Boundary Problems. Wiley, New York (1982)

6. Elliott, CM, Ockendon, JR: Weak and Variational Methods for the Moving Boundary Problems. Research Notes in Mathematics, vol. 59. Pitman, Boston (1982)

7. Barbu, V: Analysis and Control of Nonlinear Infinite Dimensional Systems. Academic Press, San Diego (1993)

8. Barbu, V: Nonlinear Semigroups and Differential Equations in Banach space. Noordhoff, Leiden (1976)

9. Lions, JL: Quelques Problèmes de la Théorie des équations non linéaires d'évolution. In: Problems in Nonlinear Analysis (C.I.M.E., Varenna, 1970), pp. 191-342. Ed. Cremonese, Rome (1971)

10. Duvaut, J, Lions, J: Sur les inéquations en mécanique et en physique. Dunod, Paris (1972)

11. Yong, J, Pan, L: Quasi-linear parabolic partial differential equations with delays in the highest order partial derivatives. J. Aust. Math. Soc. 54, 174-203 (1993)

12. Butzer, PL, Berens, H: Semi-Groups of Operators and Application. Springer, Berlin (1967)

13. Jeong, JM, Kwun, YC, Park, JY: Approximate controllability for semilinear retarded functional differential equations. J. Dyn. Control Syst. 3, 329-346 (1999)

14. Jeong, JM, Roh, HH: Approximate controllability for semilinear retarded systems. J. Math. Anal. Appl. 321, 961-975 (2006)

15. Triebel, H: Interpolation Theory, Function Spaces, Differential Operators. North-Holland, Amsterdam (1978)

16. Aubin, JP: Un théorème de compacité. C. R. Acad. Sci. 256, 5042-5044 (1963)

doi:10.1186/1029-242X-2014-387

Cite this article as: Rho and Jeong: Regularity for nonlinear evolution variational inequalities with delay terms. Journal of Inequalities and Applications 2014 2014:387.

\section{Submit your manuscript to a SpringerOpen ${ }^{\ominus}$ journal and benefit from:}

- Convenient online submission

- Rigorous peer review

- Immediate publication on acceptance

Open access: articles freely available online

- High visibility within the field

- Retaining the copyright to your article 